\title{
Análise do processo de corrosão na falha clínica de mini-implantes ortodônticos
}

\author{
Analysis of the corrosion process in the clinical failure of orthodontic miniscrew \\ Marcelo Santos BAHIA ${ }^{a}$, Ana Luiza Santiago LOPES ${ }^{\mathrm{b}}$, Paula Liparini CAETANO ${ }^{\mathrm{a}}$, \\ Robert Willer Farinazzo VITRAL ${ }^{a}$, Marcio José da Silva CAMPOS ${ }^{a \star}$ \\ ${ }^{a}$ UFJF - Universidade Federal de Juiz de Fora, Faculdade de Odontologia, Juiz de Fora, MG, Brasil \\ bUERJ - Universidade do Estado do Rio de Janeiro, Rio de Janeiro, RJ, Brasil
}

\begin{abstract}
Resumo
Introdução: Mini-implantes ortodônticos são dispositivos de ancoragem confeccionados à base de titânio, possuindo uma camada de óxido de titânio, que desempenha função importante na resistência à corrosão. Objetivo: O objetivo deste estudo foi avaliar a resistência à corrosão e a microestrutura superficial de mini-implantes novos, utilizados que foram perdidos precocemente e os que obtiveram sucesso de estabilidade, analisando se o processo de corrosão influencia a falha ou a perda de estabilidade, a fim de se comparar com as principais causas de sucesso e insucesso clínico. Material e método: A amostra foi composta por 3 grupos de 7 mini-implantes divididos da seguinte maneira: Grupo C: mini-implantes na sua forma original, como são recebidos do fabricante (controle); Grupo PE: mini-implantes utilizados em pacientes e que apresentaram perda de estabilidade precoce em até 2 meses (média de 33,5 dias); Grupo E: mini-implantes utilizados em pacientes com sucesso de estabilidade (média de 230 dias). A análise visual da superfície da rosca dos mini-implantes foi feita através de microscópio eletrônico de varredura e todos foram submetidos a ensaio de polarização cíclica potenciodinâmica em potenciostato. Resultado: A microestrutura superficial não foi significativamente alterada pela permanência e perda precoce de estabilidade em seus sítios ósseos. Conclusão: Os dados sugeriram que a corrosão não foi fator associado à falha do dispositivo ou perda de sua estabilidade, sendo outros fatores, como o tipo do mini-implante, o seu comprimento e o local de implantação, considerados mais preponderantes com influência no seu insucesso.
\end{abstract}

Descritores: Procedimentos de ancoragem ortodôntica; corrosão; ortodontia.

\begin{abstract}
Introduction: Orthodontic miniscrew are titanium-based anchoring devices, having a layer of titanium oxide, which plays an important role in corrosion resistance. Objective: The objective of this study was to evaluate the corrosion resistance and surface microstructure of new miniscrew, those who were used and lost early and those who had achieved stability success, analyzing if the corrosion process influences the failure or the loss of stability of it, in order to compare with the main causes of success and clinical unsuccess. Materials and method: The sample consisted of 3 groups of 7 miniscrews divided as follows: Group C: miniscrews in their original form, as they are received from the manufacturer (control); Group PE: miniscrews used in patients with early loss of stability in up to 2 months (mean of 33.5 days); Group E: miniscrews used in patients with stability success (mean of 230 days). The visual analysis of the screw surface of the miniscrews was done by scanning electron microscope and all were submitted to a cyclic potentiodynamic polarization in potentiostat. Result: The surface microstructure was not significantly altered by the permanence and early loss of stability in its bone sites. Conclusion: The data suggests that corrosion was not a factor associated with device failure or loss of stability, being other factors such as the type of miniscrews, its length and the site of implantation considered to be the most preponderant with influence in its failure.
\end{abstract}

Descriptors: Orthodontic anchorage procedures; corrosion; orthodontics.

\section{INTRODUÇÃO}

O mini-implante ortodôntico (MI) é um dos dispositivos mais utilizados para ancoragem ortodôntica, possui diversos tipos e desenhos, com diâmetros que variam em torno de 1,2 a 2,0 mm e comprimentos entre 6,0 e 12,0 mm, permitindo seu emprego em várias situações clínicas ${ }^{1}$.
Esses dispositivos são amplamente utilizados principalmente pela facilidade de instalação, manipulação e remoção, custo reduzido, mínima irritação aos tecidos adjacentes e possibilidade de aplicação de carga imediatamente após a instalação ${ }^{2,3}$. A versatilidade de utilização desses dispositivos fez com que novas mecânicas fossem 
desenvolvidas, influenciando a forma com que os tratamentos ortodônticos são conduzidos ${ }^{4}$.

Mini-implantes ortodônticos são confeccionados à base de titânio e, quando submetidos ao meio ácido, aos íons fluoreto ou à saliva, a proteção da camada de óxido de titânio, que desempenha função importante na resistência à corrosão, pode ser perdida e um processo de corrosão é iniciado ${ }^{5}$. A definição de corrosão é a deterioração de um material, geralmente metálico, por ação física, química ou eletroquímica do meio ambiente aliada ou não a esforços mecânicos ${ }^{6}$. Sendo que, a corrosão pode atingir todos os metais, basta haver meio corrosivo suficiente para que isso ocorra ${ }^{7}$.

A corrosão por pites é um tipo de corrosão localizada, na qual, em determinados pontos da superfície do material, o meio agressivo consegue quebrar o filme passivo (camada protetora) para depois progredir em profundidade ${ }^{7-9}$.

Os eventos de corrosão resultam na formação de pequenas cavidades na superfície do dispositivo que podem amplificar o ambiente corrosivo em torno do implante e comprometer a sua estabilidade mecânica. Isso pode levar ao encurtamento da vida útil do implante e à falha súbita ${ }^{10}$. Winters, Nutt ${ }^{11}$ demonstraram em seu estudo que a instalação do processo corrosivo por pites associado ao estresse mecânico aos quais implantes metálicos são submetidos pode acelerar a liberação de partículas e íons metálicos, levando a falhas estruturais prematuras.

O objetivo deste estudo foi avaliar a resistência à corrosão e a microestrutura superficial de mini-implantes novos, utilizados que foram perdidos precocemente e os que obtiveram sucesso de estabilidade, analisando se o processo de corrosão influencia a falha ou a perda de estabilidade, a fim de se comparar com as principais causas de sucesso e insucesso clínico.

\section{MATERIAL E MÉTODO}

A amostra foi composta de vinte e um mini-implantes ortodônticos autoperfurantes com $6 \mathrm{~mm}$ de comprimento e diâmetro de 1,5 mm, fabricados com a liga Ti6AI4V (titânio hexa-alumínio tetra-vanádio). Os mini-implantes foram divididos em 3 grupos de 7 unidades da seguinte maneira: Grupo C: mini-implantes na sua forma original, como são recebidos do fabricante (controle); Grupo PE: mini-implantes utilizados em pacientes e que apresentaram perda de estabilidade precoce em até 2 meses (média de 33,5 dias); Grupo E: mini-implantes utilizados em pacientes com sucesso de estabilidade (média de 230 dias).

Os mini-implantes utilizados no presente estudo foram inseridos em pacientes em atendimento no curso de Especialização em Ortodontia da Faculdade de Odontologia da Universidade Federal de Juiz de Fora (UFJF). O mesmo estudo foi aprovado pelo Comitê de Ética em Pesquisa da UFJF com o parecer $n^{\circ} 1.129 .481$.

Inicialmente foi realizada a análise visual da superfície da rosca dos mini-implantes através de um microscópio eletrônico de varredura (LEICA/LEO Stereoscan S440 - EMU, Rondebosch, África do Sul), equipado com detector de elétrons retroespalhados e elétrons secundários com aumento de 100 a 500 vezes. Esta análise teve como objetivo identificar alterações na superfície, como os pites, e foi realizada novamente após o ensaio de polarização nos implantes que apresentaram indícios de corrosão por pite no ensaio de polarização. Na presença de alguma imagem sugestiva de pite, foi realizada a espectrometria de energia dispersiva de raio- $X$ (EDS), para determinar os elementos químicos presentes na região.

Para a avaliação da resistência à corrosão, todos os mini-implantes foram submetidos a ensaio de polarização cíclica potenciodinâmica em potenciostato PGSTAT 204N (Metrohm Autolab ${ }^{\circledR}$ ) acoplado a um computador com o programa Advanced Electrochemical Software NOVA 2.0.

Para o ensaio de polarização, foi utilizado o esquema de três eletrodos imersos em uma solução de trabalho*. O eletrodo de trabalho foi formado pelo mini-implante acoplado, através de uma conexão, a um fio de cobre de ponta laminada com espessura de $1 \mathrm{~mm}$ dobrada para receber a cabeça do mini-implante. Para padronização da área do eletrodo de trabalho, exposto à solução de trabalho, foi desenvolvido um modelo em negativo do mini-implante em silicone de adição (Zhermack, Itália), que cobria toda a rosca do mini-implante (6 mm), deixando livre o restante da estrutura acoplada ao fio de cobre para isolamento com silicone. O contraeletrodo é um eletrodo de platina de grande área que atuou como um catodo recebendo os elétrons liberados pelo mini-implante (anodo). O terceiro eletrodo foi o de $\mathrm{Ag} / \mathrm{AgCl} 3$ Molar que funcionou como eletrodo de referência. A célula eletroquímica onde os ensaios foram realizados possuía lugares padronizados para inserção de cada eletrodo, garantindo manutenção da distância entre eles em todos os experimentos.

Os eletrodos foram imersos em $50 \mathrm{~mL}$ de solução de ringer lactato $(0,3 \mathrm{~g} / 100 \mathrm{~mL}$ de lactato de sódio, $0,6 \mathrm{~g} / 100 \mathrm{~mL}$ de cloreto de sódio, 0,03 g/100 mL de cloreto de potássio e 0,02 g/100 mL de cloreto de cálcio). A solução de ringer lactato foi trocada a cada experimento a $37^{\circ} \mathrm{C}+-1^{\circ} \mathrm{C}$, e a temperatura foi controlada através de Banho Ultratermostático (SL 152, Solab, Piracicaba, SP).

O OCP foi determinado previamente a cada ensaio quando se detectava pequena variação do potencial em função do tempo $(\Delta \mathrm{E} \Delta \mathrm{t})$, variação está próxima de zero. Os ensaios de polarização cíclica tiveram como potencial inicial o OCP, aumentando a uma taxa de $0,33 \mathrm{mV} / \mathrm{s}(1,2 \mathrm{~V} / \mathrm{h})$ e tendo como potencial final $1,4 \mathrm{~V}$ em relação ao OCP. O potencial final foi pré-determinado em 1,4 V porque, após esse potencial, em ensaios com a liga Ti6Al4V, o que se observa são processos de dissociação do oxigênio ${ }^{8}$.

A partir da curva de polarização, foram registradas medidas referentes ao OCP (potencial de circuito aberto), ipp (corrente de passivação primária, considerada o ponto final de corrente no trecho de passivação) e Epp (potencial de passivação primário, considerado o ponto final de potencial no trecho de passivação).

Os procedimentos de polarização foram realizados no interior de uma gaiola de Faraday, com o objetivo de isolar o sistema das ondas eletromagnéticas externas, evitando interferências e possibilitando um resultado mais fidedigno. Ao final do ensaio de polarização, os procedimentos de limpeza e armazenamento dos mini-implantes foram repetidos, a fim de prepará-los para a análise de superfície.

\footnotetext{
Costa TD. Avaliação da resistência à corrosão das limas endodônticas Protaper Universal, MTWO, RECIPROC e Wave-One imersas em solução de hipoclorito de sódio a 2,5\%. Juiz de Fora: Universidade Federal de Juiz de Fora; 2014.
} 


\section{Análise Estatística}

Foi realizado o teste de normalidade de Shapiro-Wilk para avaliar o padrão de distribuição da amostra, seguida dos testes de Mann-Whitney para as variáveis previamente identificadas como de distribuição não normal e do teste t de Student para amostras independentes para a variável identificada com distribuição normal. O nível de significância adotado foi de $\alpha=0,05$ e todos os testes foram realizados através do software SPSS Statistics 20.0 (SPSS, Chicago, IL, EUA).

\section{RESULTADO}

\section{Caracterização Visual Superficial da Rosca Através do MEV}

As imagens obtidas dos mini-implantes do grupo controle demonstraram, de maneira geral, superfície polida e regular, sem presença de manchas ou partículas aderidas (Figura 1).

No mini-implante de número 3, foi detectada uma cavidade de profundidade maior que seu diâmetro, sugerindo uma corrosão por pite (Figura 2). Foi realizada a EDS da cavidade à superfície adjacente, sendo constatado processo de corrosão do Ti na região, com redução da concentração de Ti de $83,22 \%$ na região sem porosidade em comparação à concentração de Ti de 32,45\% na região com porosidade e consequente acréscimo das concentrações do Al e V. Dessa forma, pode-se afirmar que a porosidade encontrada na MEV é uma cavidade do tipo pite.
Foi possível visualizar imperfeições superficiais, em formatos positivos e negativos, característicos do processo de fabricação do dispositivo nos três grupos.

\section{Análises das Curvas de Polarização Cíclica}

O teste de normalidade de Shapiro-Wilk mostrou que apenas a variável OCP poderia ser considerada como oriunda de uma população com distribuição normal. As demais variáveis (Epp, ipp e tOCP) não satisfazem o pressuposto de normalidade $(\mathrm{p}<0,05)$ em pelo menos um dos grupos. Por esse motivo, utilizou-se o teste $t$ de Student para amostras independentes apenas para a variável OCP e o teste não paramétrico de Mann-Whitney para as demais variáveis.

Ao analisar os grupos de mini-implantes dois a dois, não foi encontrada diferença estatisticamente significativa para as variáveis tOCP, OCP, Epp e ipp (Tabela 1).

As curvas de polarização obtidas para os mini-implantes avaliados nos três grupos foram muito semelhantes, não havendo em nenhuma delas aumento repentino de corrente, o que poderia caracterizar corrosão por pite e quebra da resistência à corrosão (Figura 3A). Exceção do mini-implante número 3 do Grupo Controle (grupo 1), que apresentou um aumento repentino de corrente medida no potencial de $0,80 \mathrm{~V}$, caracterizando a quebra da resistência à corrosão do material e o início da corrosão por pite (Figura 3B).
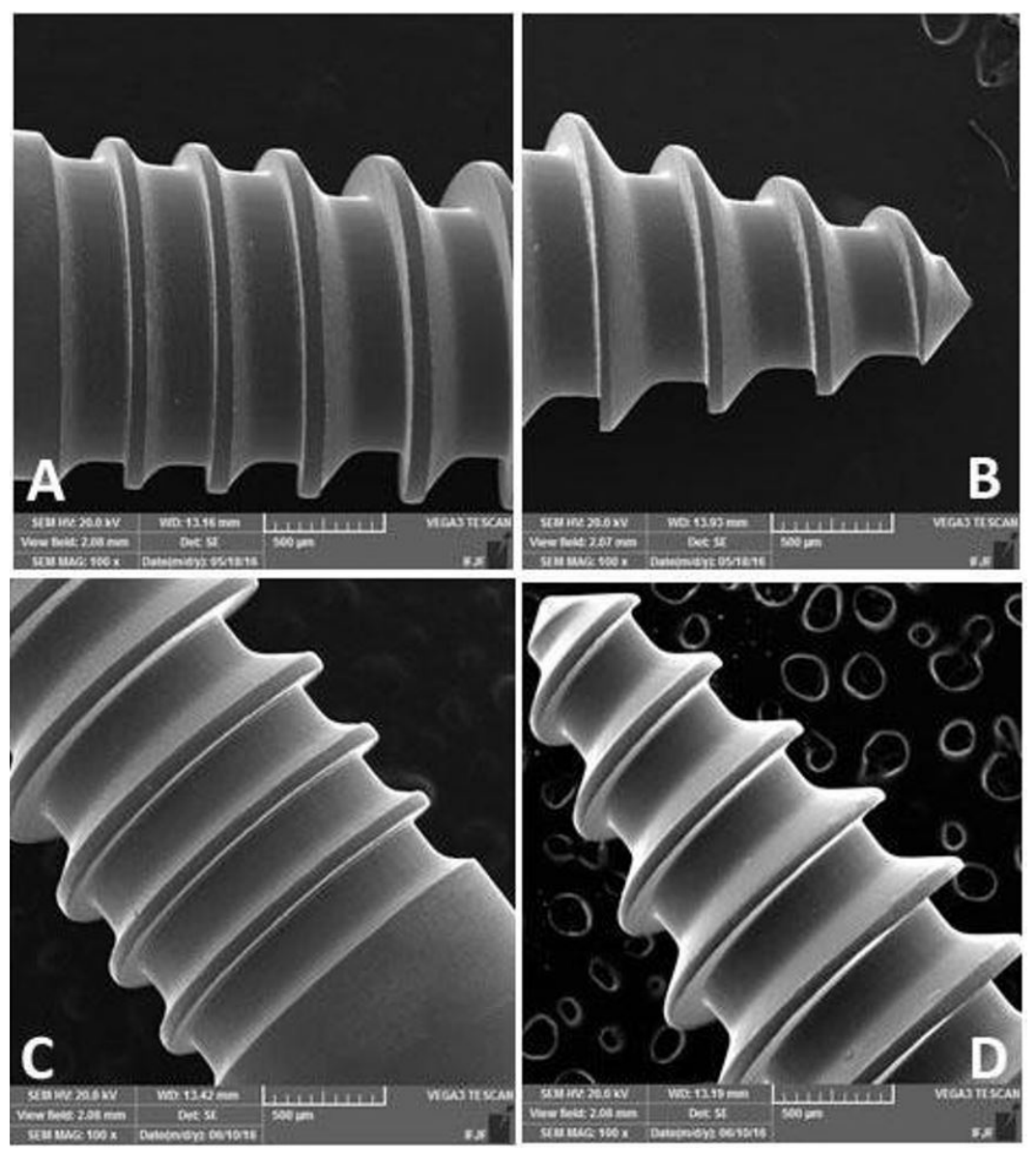

Figura 1. Imagem da microscopia eletrônica de varredura do mini-implante número 1 do Grupo Controle (Grupo 1) com aumento de 500 vezes, realizada antes (A - corpo e B - ponta) e após (C - corpo e D - ponta) ensaio de polarização. 


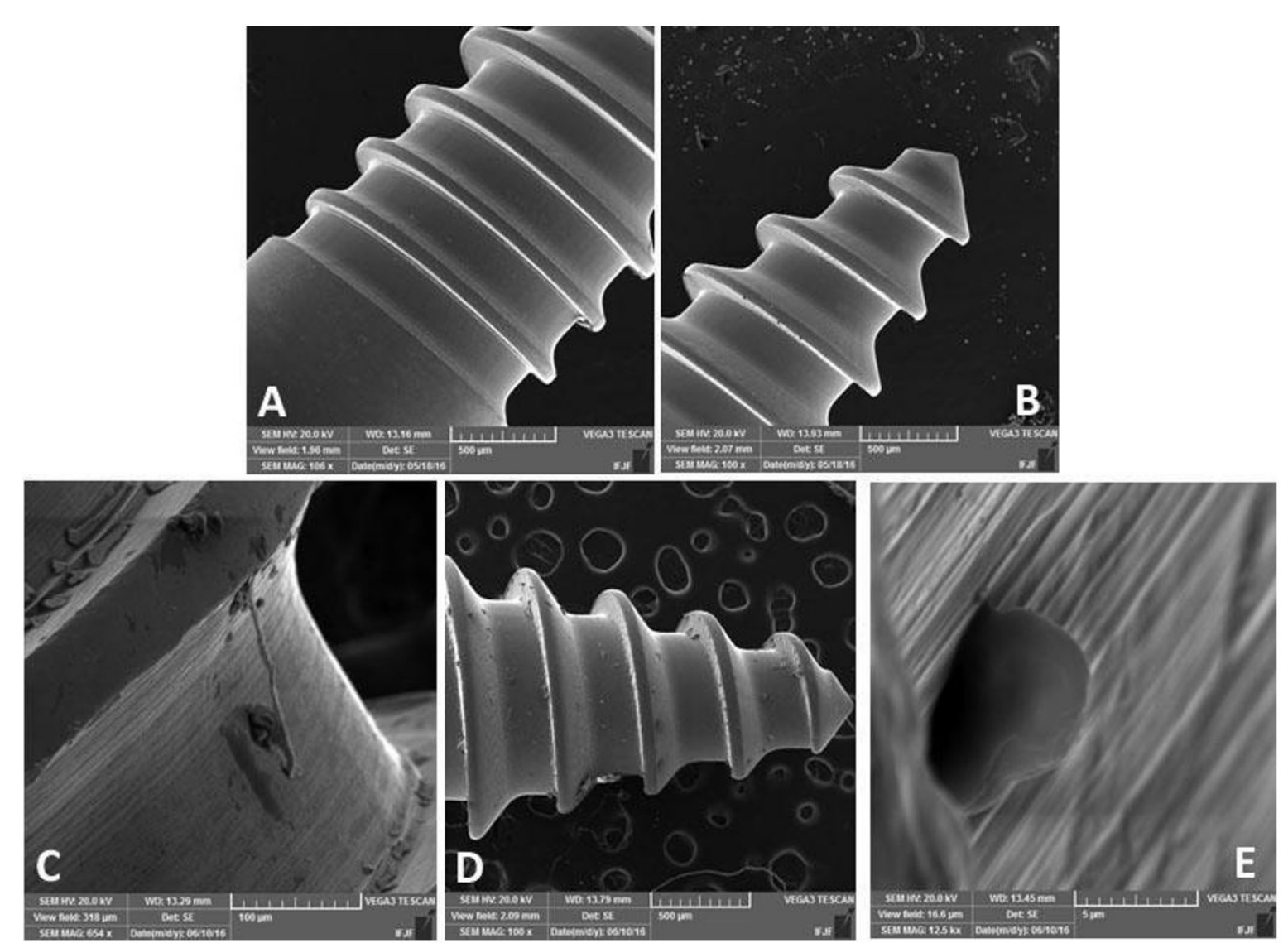

Figura 2. Imagem da microscopia eletrônica de varredura do mini-implante número 3 do Grupo Controle (Grupo 1) com aumento de 500 , 100 e $5 \mu \mathrm{m}$ vezes, realizada antes (A - corpo e B - ponta) e após (C - corpo, D - ponta e E - pite) o ensaio de polarização.
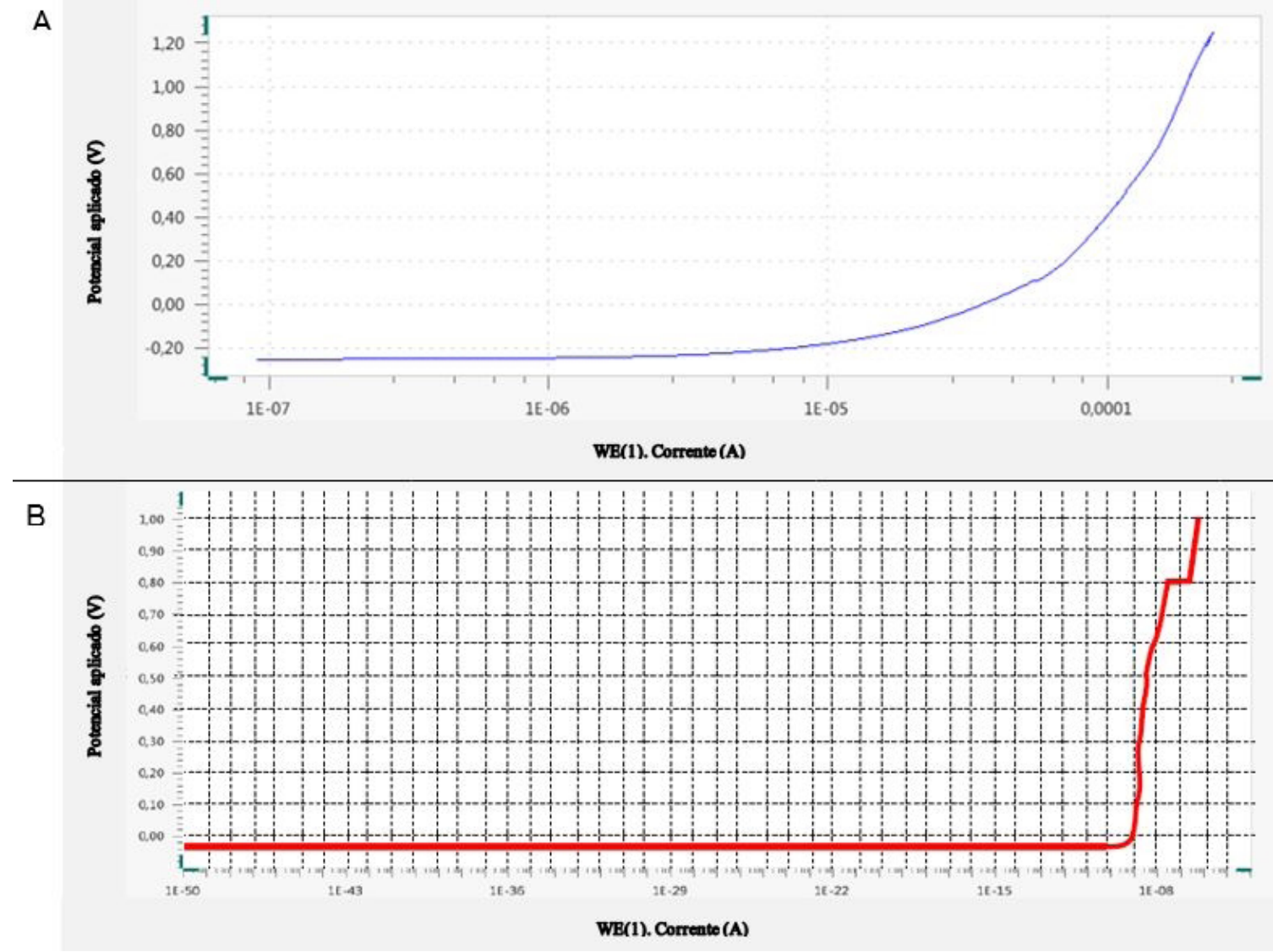

Figura 3. A - Curva de polarização obtida com o ensaio de polarização do mini-implante do Grupo Controle (Grupo 1); B - Curva de polarização obtida com o ensaio de polarização do mini-implante 3 do Grupo Controle, evidenciando Epite em 0,80 V. 
Tabela 1. Média e desvio padrão das variáveis OCP, tOCP, Epp e ipp dos grupos C, PE e E* teste t para amostras independentes

\begin{tabular}{|c|c|c|c|c|c|c|c|c|c|}
\hline & \multicolumn{2}{|c|}{$\begin{array}{l}\text { Controle } \\
\text { (C) }\end{array}$} & \multicolumn{2}{|c|}{$\begin{array}{c}\text { Perda de Estabilidade } \\
\text { (PE) }\end{array}$} & \multicolumn{2}{|c|}{$\begin{array}{l}\text { Estável } \\
\text { (E) }\end{array}$} & \multicolumn{3}{|c|}{$\begin{array}{c}\text { Comparação } \\
(\mathbf{p} \text {-valor })^{\star}\end{array}$} \\
\hline & Média & DP & Média & DP & Média & DP & $C \times P E$ & $\mathrm{CxE}$ & $P E \times E$ \\
\hline $\mathrm{OCP}(\mathrm{V})$ & $-0,455$ & 0,221 & $-0,461$ & 0,197 & $-0,579$ & 0,185 & 0,958 & 0,276 & 0,269 \\
\hline T OCP (s) & 676,603 & 183,214 & 733,207 & 89,943 & 743,998 & 202,008 & 0,477 & 0,526 & 0,899 \\
\hline Epp (v) & 0,999 & 0,205 & 1,025 & 0,195 & 0,888 & 0,195 & 0,791 & 0,334 & 0,214 \\
\hline $\operatorname{ipp}\left(\mu \mathrm{A} / \mathrm{cm}^{2}\right)$ & 51,589 & 24,829 & 40,336 & 29,720 & 38,697 & 22,386 & 0,457 & 0,328 & 0,909 \\
\hline
\end{tabular}

\section{DISCUSSÃO}

A taxa de insucesso global dos mini-implantes varia entre $7 \%$ e $19 \%^{12-15}$, sendo que o risco de falha é maior durante os primeiros 100 dias, diminuindo gradualmente ${ }^{12}$. Isso corrobora os autores, pois o Grupo PE apresentou um tempo médio de 33,5 dias de permanência em seus sítios de inserção. Yi Lin et al. ${ }^{14}$ relataram um período médio de 3,5 meses para a falha dos mini-implantes após o carregamento.

O tipo de mini-implante ${ }^{16}$, o comprimento do dispositivo ${ }^{14,15,17}$, o local de implantação e as características do tecido mole em torno do $\mathrm{MI}^{16}$ são fatores significativos na falha.

Foi analisado, no presente estudo, se a resistência à corrosão pode ser associada à falha do dispositivo de ancoragem. Segundo Gittens et al. ${ }^{10}$, os eventos de corrosão resultam na formação de pequenas cavidades na superfície do dispositivo que podem amplificar o ambiente corrosivo em torno do implante e comprometer a sua estabilidade mecânica. Isso pode levar ao encurtamento da vida útil do implante e à falha súbita. Winters, Nutt ${ }^{11}$ demonstraram em seu estudo que a instalação do processo corrosivo por pites associado ao estresse mecânico aos quais implantes metálicos são submetidos pode acelerar a liberação de partículas e íons metálicos, levando a falhas estruturais prematuras.

No ensaio de polarização, o meio eletrolítico deve mimetizar o meio em que o material analisado fica em contato, simulando o processo eletroquímico que ocorre com determinada amostra. O presente estudo almejou avaliar o processo eletroquímico que ocorre no corpo do mini-implante, o qual fica inserido no osso do paciente, por esta razão, o meio eletrolítico de escolha foi o ringer lactato, por ser isotônico ao plasma sanguíneo. Outras soluções foram utilizadas para simular o fluido corpóreo, como a solução de $\operatorname{Merck}\left(\mathrm{NaCl} \mathrm{mol} \mathrm{L}{ }^{-1}\right)^{8}$, solução salina tamponada ${ }^{18}$ e solução de $\mathrm{NaCl}$ 0,9 $\mathrm{mol} \mathrm{L}^{-1}$. A saliva artificial Fusayama também foi utilizada em outras pesquisas ${ }^{2,5,19}$, porém esta escolha só faria sentido se a cabeça do mini-implante fosse o objetivo do estudo, uma vez que, em sua aplicação clínica, esta é a única parte do mini-implante em contato com a saliva.

Como o ensaio de polarização mede a corrente de elétrons do eletrodo de trabalho (anodização), correntes elétricas oriundas de ondas eletromagnéticas externas podem modificar as condições eletromagnéticas da célula eletroquímica, influenciando a corrente de elétrons no interior desta célula e, consequentemente, comprometendo todo o ensaio de polarização ${ }^{20}$. Para evitar tais alterações, todos os ensaios de polarização desta pesquisa foram realizados no interior de uma Gaiola de Faraday ligada ao aterramento do sistema elétrico. As demais pesquisas referenciadas ${ }^{2,5,8,18,19}$ não a mencionaram e/ou não utilizaram a Gaiola de Faraday em seus experimentos, o que poderia gerar dúvidas quanto aos resultados obtidos.

O potencial de circuito aberto (OCP) é definido como o potencial de um material condutor de elétrons imerso em um meio eletrolítico condutor de íons e medido em relação a um eletrodo de referência, sendo sua redução associada à predisposição à corrosão ${ }^{2}$. Como não houve diferença estatisticamente significativa para os valores de OCP entre os grupos avaliados na presente pesquisa, os resultados indicam que a grande reatividade química dos mini-implantes não foi associada à perda precoce.

Em relação, ao mini-implante de número 3 do Grupo C, que apresentou cavidade do tipo pite, seu OCP foi de $-0,045 \mathrm{~V}$, bem abaixo da média de seu grupo, confirmando a elevada reatividade associada à presença do processo corrosivo.

Sabe-se que a estabilidade da camada passiva do material é dependente do potencial eletrolítico e das agressões químicas que o material sofre ${ }^{21}$, podendo ser alterada pela presença de íons cloreto no plasma sanguíneo ${ }^{9}$, pelo $\mathrm{pH}$ do fluido bucal, pela presença de processos inflamatórios e pela disponibilidade de oxigênio $^{21}$. Como os mini-implantes do Grupo C não tiveram sua camada passiva agredida pelos processos de inserção, permanência, utilização e remoção, a estabilização do potencial foi mais rápida, o que é refletido por um tOCP do grupo controle 9\% menor que os dos grupos de mini-implantes que foram utilizados em pacientes (Grupo PE e Grupo E).

As médias do Epp e do ipp encontradas foram de $0,996 \mathrm{~V}$ e de $51,589 \mu \mathrm{A} / \mathrm{cm}^{2}$ no Grupo C; $1,025 \mathrm{~V}$ e de $40,336 \mu \mathrm{A} / \mathrm{cm}^{2}$ no Grupo PE; e $0,888 \mathrm{~V}$ e de $38,697 \mu \mathrm{A} / \mathrm{cm}^{2}$ no Grupo E, respectivamente. Quanto menor o valor de Epp e de ipp, menor a taxa de corrosão do filme passivo e, consequentemente, maior o seu poder protetor ${ }^{* *}$. Logo, como não houve diferença estatisticamente significativa entre os grupos, com base na presente pesquisa, pode-se afirmar que tanto os mini-implantes que sofreram perda precoce, quanto os que obtiveram sucesso de estabilidade, quanto os MI na sua forma original recebidos de fábrica apresentaram o mesmo poder protetor contra corrosão. Assim, a falha observada em até 2 meses não foi atribuída à diferença de resistência à corrosão.

\footnotetext{
** Rodriguez RMHP. Formação de óxidos nos revestimentos de alumínio depositados por aspersão térmica. Curitiba: Universidade Federal do Paraná; 2003.
} 
No presente estudo, os mini-implantes do Grupo C não apresentaram alteração ao serem analisados pelo MEV, tendo sua superfície regular e sem sinal de corrosão por pite, além disso apresentaram bordas nítidas, bem definidas e sem risco. Os Grupos E e PE exibiram discreta alteração de superfície evidenciando algumas manchas, sem alteração de bordas. Tais manchas podem ser atribuídas ao processo de polarização que ocorreu nos MIs testados. El-Wassefy et al. ${ }^{22}$ afirmaram que os mini-implantes podem apresentar bordas mais suaves associadas aos processos de inserção e de remoção. Em um estudo realizado com mini-implantes removidos do osso e analisados por meio do $\mathrm{MEV}$, estes não mostraram defeitos ou imagem sugestiva de corrosão, mas mostraram a superfície mais lisa, a ponta mais arredondada e riscos superficiais, quando comparados a MIs novos, sugerindo ter havido desgaste em sua superfície ${ }^{* * *}$. Uma justificativa para as bordas na pesquisa em questão serem iguais entre os grupos e as demais pesquisas observarem essa diferença pode ser o fato de a inserção e remoção ter ocorrido de forma mais branda na amostra testada.

Apesar da determinação prévia de um potencial máximo de 1,4 V para as polarizações, nenhum experimento atingiu tal potencial

\footnotetext{
*** Mattos CT. Avaliação da morfologia superficial e propriedades mecânicas de mini-implantes removidos da cavidade bucal e de mini-implantes autoclavados. Rio de Janeiro: Universidade Federal do Rio de Janeiro; 2010.
}

espontaneamente, sendo a média do maior potencial (Epp) de 0,996 V e 1,025 V para o grupo 1 e para o grupo 2, respectivamente. Outros estudos utilizaram limites menores $\left(700 \mathrm{mV}^{19}, 400 \mathrm{mV}^{18}\right)$ ou maiores $\left(1,8 \mathrm{~V}^{5} ; 2 \mathrm{~V}^{2}\right)$. Entretanto, para a liga em questão, após esse valor de $1,4 \mathrm{~V}$, o que se observa são processos de dissociação do oxigênio ${ }^{8}$, sem relevância para o que foi proposto.

\section{CONCLUSÃO}

A microestrutura superficial não foi significativamente alterada pela permanência e perda precoce de estabilidade em seus sítios ósseos. Os dados sugeriram que a corrosão não foi fator associado à falha do dispositivo ou perda de sua estabilidade, sendo outros fatores, como o tipo do mini-implante, o seu comprimento e o local de implantação, considerados mais preponderantes com influência no seu insucesso.

\section{AGRADECIMENTOS}

Os autores agradecem à Fundação de Amparo à Pesquisa de Minas Gerais (FAPEMIG) por ter financiado esta pesquisa.

\section{REFERÊNCIAS}

1. Asscherickx K, Vannet BV, Wehrbein H, Sabzevar MM. Success rate of miniscrews relative to their position to adjacent roots. Eur J Orthod. 2008 Aug;30(4):330-5. http://dx.doi.org/10.1093/ejo/cjn019. PMid:18632839.

2. Souza JCM, Henriques M, Teughels W, Ponthiaux P, Celis JP, Rocha LA. Wear and corrosion interactions on titanium in oral environment: literature review. J Bio Tribo Corros. 2015;1(2):13. http://dx.doi.org/10.1007/s40735-015-0013-0.

3. Cordeiro JM, Barão VAR. Is there scientific evidence favoring the substitution of commercially pure titanium with titanium alloys for the manufacture of dental implants? Mater Sci Eng C. 2017 Feb;1(71):1201-15. http://dx.doi.org/10.1016/j.msec.2016.10.025. PMid:27987677.

4. Schätzle M, Männchen R, Zwahlen M, Lang NP. Survival and failure rates of orthodontic temporary anchorage devices: a systematic review. Clin Oral Implants Res. 2009;20(12):1351-9. http://dx.doi.org/10.1111/j.1600-0501.2009.01754.x. PMid:19793320.

5. Barão VAR, Mathew MT, Assuncão WG, Yuan JC-C, Wimmer MA, Sukotjo C. Stability of cp-Ti and Ti-6Al-4V alloy for dental implants as a function of saliva pH - an electrochemical study. Clin Oral Implants Res. 2012 May;23(9):1055-62. http://dx.doi.org/10.1111/j.16000501.2011.02265.x. PMid:22092540.

6. Gentil V. Corrosão. Rio de Janeiro: LTC-Livros Técnicos e Científicos; 1996.

7. Olmedo DG, Tasat DR, Duffó G, Guglielmotti MB, Cabrini RL. The issue of corrosion in dental implants: a review. Acta Odontol Latinoam. 2009 Abr;22(1):3-9. PMID: 19601489.

8. Ramires I, Guastaldi AC. Estudo do biomaterial Ti-6Al-4V empregando-se técnicas eletroquímicas e XPS. Quim Nova. 2002 Jul;25(1):10-4. http://dx.doi.org/10.1590/S0100-40422002000100003.

9. Silva EF, Oliveira LFC. Caracterização química e metalográfica dos aços inoxidáveis de implantes removidos de pacientes. Acta Ortop Bras. 2011;19(5):280-5. http://dx.doi.org/10.1590/S1413-78522011000500003.

10. Gittens RA, Olivares-Navarrete R, Tannenbaum R, Boyan BD, Schwartz Z. Electrical implications of corrosion for osseointegration of titanium implants. J Dent Res. 2011 Dec;90(12):1389-97. http://dx.doi.org/10.1177/0022034511408428. PMid:21555775.

11. Winters G, Nutt M. STP1438-EB: Stainless Steels for Medical and Surgical Applications. West Conshohocken: ASTM International; 2003. https://doi.org/10.1520/STP1438-EB.

12. Manni A, Cozzani M, Tamborrino F, De Rinaldis S, Menini A. Factors influencing the stability of miniscrews. A retrospective study on 300 miniscrews. Eur J Orthod. 2011 Aug;33(4):388-95. http://dx.doi.org/10.1093/ejo/cjq090. PMid:20926556.

13. Dalessandri D, Salgarello S, Dalessandri M, Lazzaroni E, Piancino M, Paganelli C, et al. Determinants for success rates of temporary anchorage devices in orthodontics: a meta-analysis (n > 50). Eur J Orthod. 2014 Jul;36(3):303-13. http://dx.doi.org/10.1093/ejo/cjt049. PMid:23873818.

14. Yi Lin S, Mimi Y, Ming Tak CM, Kelvin Weng Chiong F, Hung Chew W. A study of success rate of miniscrew implants as temporary anchorage devices in Singapore. Int J Dent. 2015 Mar;2015:294670. http://dx.doi.org/10.1155/2015/294670. PMid:25861272.

15. Melo ACM, Andrighetto AR, Hirt SD, Bongiolo ALM, Silva SU, Silva MAD. Risk factors associated with the failure of miniscrews - A tenyear cross sectional study. Braz Oral Res. 2016;30(1):e124. http://dx.doi.org/10.1590/1807-3107BOR-2016.vol30.0124. PMid:27783770. 
16. Yao C-CJ, Chang H-H, Chang JZ-C, Lai H-H, Lu S-C, Chen Y-J. Revisiting the stability of mini-implants used for orthodontic anchorage. J Formos Med Assoc. 2015 Nov;114(11):1122-8. http://dx.doi.org/10.1016/j.jfma.2014.08.001. PMid:25260551.

17. Romano FL, Consolaro A. Why are mini-implants lost: the value of the implantation technique! Dental Press J Orthod. 2015 Feb;20(1):23-9. http://dx.doi.org/10.1590/2176-9451.20.1.023-029.oin. PMid:25741821.

18. Manhabosco TM, Muller IL, Santos CB. Tribocorrosão da liga TI6AL4V em solução salina tamponada com fosfato. Quim Nova. 2009;32(9):22637. http://dx.doi.org/10.1590/S0100-40422009000900004.

19. Knutson KJ, Berzins DW. Corrosion of orthodontic temporary anchorage devices. Eur J Orthod. 2013 May;35(4):500-6. http://dx.doi. org/10.1093/ejo/cjs027. PMid:22573910.

20. Risos A, Long N, Hunze A, Gouws G. A 3D Faraday shield for interdigitated dielectrometry sensors and its effect on capacitance. Sensors (Basel). 2016;17(1):77-90. http://dx.doi.org/10.3390/s17010077. PMid:28042868.

21. Fage SW, Muris J, Jakobsen SS, Thyssen JP. Titanium: a review on exposure, release, penetration, allergy, epidemiology, and clinical reactivity. Europ Env Cont Dermat Res. 2016 Feb;74(6):323-45. http://dx.doi.org/10.1111/cod.12565. PMid:27027398.

22. El-Wassefy N, El-Fallal A, Taha M. Effect of different sterilization modes on the surface morphology, ion release, and bone reaction of retrieved micro-implants. Angle Orthod. 2015 May;85(1):39-47. http://dx.doi.org/10.2319/012014-56.1. PMid:24866836.

\section{CONFLITOS DE INTERESSE}

Os autores declaram não haver conflitos de interesse.

\section{*AUTOR PARA CORRESPONDÊNCIA}

Marcio José da Silva Campos, UFJF - Universidade Federal de Juiz de Fora, Faculdade de Odontologia, Rua José Lourenço Kelmer, s/n, Campus Universitário, Bairro Martelos, 36036-300 Juiz de Fora - MG, Brasil, e-mail: marcio.campos@uff.edu.br

Recebido: Dezembro 18, 2018 Aprovado: Janeiro 17, 2019 